\title{
Civilisations
}

Revue internationale d'anthropologie et de sciences

humaines

44 | 1997

Les peuples des forêts tropicales

\section{Essartage et confusionnisme}

À propos des Mnong Gar du Vietnam Central

\section{Georges Condominas}

\section{(2) OpenEdition}

12 Journals

Édition électronique

URL : http://journals.openedition.org/civilisations/1634

DOI : 10.4000/civilisations. 1634

ISSN : 2032-0442

Éditeur

Institut de sociologie de l'Université Libre de Bruxelles

\section{Édition imprimée}

Date de publication : 1 janvier 1997

Pagination : 228-237

ISBN : 2-87263-122-4

ISSN : 0009-8140

\section{Référence électronique}

Georges Condominas, «Essartage et confusionnisme », Civilisations [En ligne], 44 | 1997, mis en ligne le 29 juin 2009, consulté le 10 décembre 2020. URL : http://journals.openedition.org/civilisations/1634 ; DOI : https://doi.org/10.4000/civilisations.1634

(c) Tous droits réservés 
- ESSARTRGE ET CONFUSIONNISME:

À propos des Mnong Gar

du Vietnam Central

Georges CONDOMWMAS 
Permettez-moi tout d'abord de vous remercier pour m'avoir invité à parler d'amis à qui je dois tant, les Hommes de la Forêt (Pbii Brêe) comme leurs voisins aux rizières permanentes appellent les Mnong Gar.

Il y a une vingtaine d'années quelqu'un m'avait envoyé de Bruxelles la première page du journal Le Soir, surprise! L'éditorial signé par un académicien était intitulé «Nous avons mangé la forêt», titre de mon premier livre, une chronique relatant la vie quotidienne d'un village Mnong. J'ai été très flatté. J'ai lu l'article. Oh horreur! ou bien l'académicien en question n'avait rien compris, ou bien, et je crois que c'est cela qui s'est passé, il n'avait pas lu mon livre. Ce qui est très vexant! Dès qu'on tient une plume, on se figure que ce qu'on écrit est important, et si on découvre que quelqu'un vous cite sans vous avoir lu, alors on est horriblement vexé.

Bien sûr cela n'arrive jamais à des gens qui ont des gros tirages, mais hélas ce n'est pas mon cas! Alors, pourquoi cette réaction! Parce que l'académicien vitupérait, indigné : «C'est un scandale! Partout sur cette terre on détruit les forêts! Les primitifs eux-mêmes; par exemple les Mnong; ils mangent la forêt, etc...». Il n'avait donc rien compris. Ou bien, il s'était arrêté au titre et n'avait même pas lu les deux premières pages du livre. Sans doute se voulait-il écologiste. En fait, il semble qu'il ignorait ce qu'est un équilibre écologique et n'avait pas vu que les Mnong s'ils mangent la forêt, ne la détruisent pas : celle qu'ils ont abattue, ils la laissent se régénérer des années durant et ils ne reviennent au même endroit que beaucoup plus tard, lorsque de beaux arbres ont poussé, ont pris la place de ceux qu'ils avaient "mangé» dix ou vingt ans auparavant. Si seulement il avait lu la courte introduction, une chose aurait accroché son attention : les Mnong en employant une expression liée à l'espace, indiquaient une date, ils utilisaient une donnée spatiale pour exprimer un fait temporel; il aurait constaté que ces usauvages" n'avaient pas attendu Minkowski et Einstein pour avoir la notion d'espace-temps. Je vous ai livré cette anecdote seulement pour montrer à quel point il est injuste d'évaluer de haut une culture selon les critères de la nôtre; celle des forestiers entre autres doit être placée dans son contexte culturel.

Le leur a pour cadre général l'Asie du SudEst. Vous avez là un ensemble de sociétés enchevêtrées - ou plutôt d'espaces sociaux (au sens où j'ai utilisé ce concept qui présente l'avantage de considérer les groupes dans leur dynamique spatio-temporelle). Cette région du monde présente une variété considérable d'espaces sociaux. Cela va des nomades forestiers de Malaisie que Françoise Aubaile-Sallenave (Introduction régionale, ce volume) a évoqués et qui sont de tout petits groupes qui circulent dans un vaste territoire de forêts, des cueilleurs - pour moi la chasse et la pêche entrent dans les «industries de cueillette» comme dirait Marcel Mauss - jusqu'aux grands empires, que ce soit l'Empire vietnamien inspiré du modèle chinois, ou l'Empire angkorien qui a été l'un des plus grands empires qui aient existé et qui a été très influencé par la civilisation indienne, mais n'en demeure pas moins une civilisation originale, la civilisation khmère.

Des uns aux autres, les rapports à la forêt, ou si vous voulez l'exploitation de la forêt, est totalement différente. Laissons de côté les grands 
espaces sociaux que je viens de mentionner et qui sortent de notre propos. Prenons par exemple celui des cueilleurs-pêcheurs-chasseurs que sont les Phi Tong Luang (c'est-à-dire les «Esprits des Feuilles Jaunes») comme les appellent les Lao et les Siamois, ils vivent de la forêt sans y toucher, car ils n'ont aucune activité agricole. Par ailleurs, nous avons aussi parmi les petits espaces sociaux, des essarteurs, comme les Mnong Gar, qui défrichent un pan de forêt, laissent les abattis sécher sur place et, juste avant la saison des pluies, y mettent le feu, sèment sur le sol enrichi de cendres, puis sarclent les mauvaises herbes et, lorsque le paddy a mûri, moissonnent. C'est ce qu'ils appellent «manger la forêt». Ils mangent le riz qui a poussé sur le sol de cette forêt dont l'humus et les cendres ont fourni un engrais naturel. Ils ne reviennent sur le même site que dix à vingt ans plus tard. Lorsque vous passez dans une

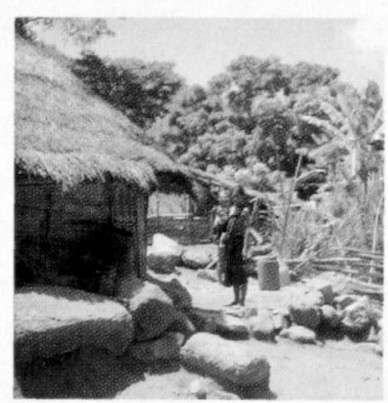
forêt qui a subi l'essartage une ou deux décennies plus tôt, vous pouvez admirer des arbres aux troncs magnifiques! Les Mnong ne détruisent pas pour détruire. Ils parlent aux arbres, aux biefs, aux rochers, aux cerfs et aux oiseaux. Un lien très étroit existe entre l'homme et la nature. On ne tue pas les animaux pour le plaisir de tuer : la chasse reste limitée au gibier qu'on peut consommer. Au cours des siècles et des millénaires, les ancêtres ont observé attentivement «les us et coutumes» des êtres qui comme eux-mêmes, peuplent cet environnement et ont légué à ces forestiers en héritage une connaissance intime de leur milieu, à la fois réaliste et colorée d'un bel imaginaire poétique (mais n'estce pas cela le réalisme?). Les Mnong ne suivent aucune doctrine d'action, mais agissent avec un flair, un sens de l'équilibre naturel. Ils sont simplement animés d'un sentiment profond d'accord avec le milieu, où tous les êtres, plantes et animaux, monts et eaux, possèdent une âme comme eux-mêmes.

Quels sont les rapports de ces nomades et semi-nomades avec les autres groupes? Ils ne faut pas croire que ces forestiers sont restés totalement isolés; même si jusqu'à une époque récente à l'échelle de l'histoire, leurs relations avec les sédentaires riziculteurs ont été marquées de crainte et de méfiance, elles n'en existaient pas moins, limitées, il est vrai, à des échanges de biens! Ils fournissaient des produits de la forêt tels que ivoire, bois de cerf, plantes rares médicinales, aux gens des plaines; ces derniers leur donnant en contrepartie sel, gongs et jarres. Ces «biens de valeur» tiennent une importance considérable dans leur culture. Il y avait aussi des captures d'esclaves, soit par les Montagnards lorsqu'ils descendaient razzier les plaines en bordure des plateaux, soit, chez eux, par les expéditions de Vietnamiens, de Lao ou de Cambodgiens. Françoise Aubaille-Sallenave a signalé l'importance du rotin pour les cueilleurschasseurs comme produits d'échanges actuels. J'ai eu l'occasion de le constater personnellement en Malaisie chez les Semang et en Inde chez les Cholonaikan des falaises de Nilgiri; dans ces pays, les forestiers en question sont complètement sous 
la dépendance de membres du groupe dominant qui exploitent commercialement le rotin collecté par les Aborigènes.

$\grave{\lambda}$ propos d'essartage, je ne suivrai pas Michael Rössler (ce volume) lorsqu il dit préférer «agriculture itinérante sur brûlis» à «essartage» et shifting cultivation à swidden. Pour moi, sémantiquement, le mot "essart", comme le mot swidden, contient en lui-même tous les aspects de ce type d'agriculture. D'autre part, si en Afrique on peut encore utiliser l'ancienne expression «agriculture itinérante sur brûlis», d'ailleurs très descriptive, il vaut mieux l'éviter en Asie du Sud-Est. Pourquoi? parce que «agriculture itinérante» fait immédiatement surgir dans l'esprit des responsables locaux l'image du nomade, peu appréciée, et "sur brûlis", celle du feu, terrifiante, chez les gens des plaines; au fond dans toutes les civilisations sédentaires, voilà une double image qui provoque une vraie répulsion : des hommes qui vadrouillent constamment en forêt et qui de surcroît n'hésitent pas à y mettre le feu. Des êtres instables, enfants des quatre vents, paresseux (ils fuient le vrai travail), incendiaires par-dessus le marché : la vision même qu'on se fait du vrai «sauvage», du moï. Consultez les vieux ouvrages d'exploration, vous trouverez des termes très péjoratifs tels que Mö̈, Kha, Phnong employés par les Vietnaniens, les Lao et les Khmers pour désigner les gens de la forêt; ils ont été repris tels quels comme ethnonymes par les explorateurs. En outre, un même groupe ethnique à cheval sur

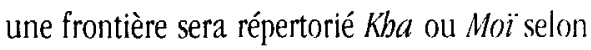
l'endroit où il se trouve. D'où le terme de ProtoIndochinois, assez laid je l'avoue, mais que par commodité j'ai calqué sur Proto-Malais alors tout à fait courant dans le monde insulindien, car que vous alliez chez les Dayak de Bornéo-Kalimantan, ou chez les Mnong ou les Rhadés, vous avez affaire au même large complexe culturel. Proto-Malais, Proto-Indochinois, vous parlez des uns et vous évoquez les autres. Il y a chez eux une variété extraordinaire d'essartages allant de pratiques simples (mais non rudimentaires) à des techniques très élaborées et hautement productives, mais non une seule forme d'allure primitive, cette caricature dont parlent les gens des basses terres.

Cette vision grotesque de l'autre, d'où vientelle? Ce rejet de l'autre par les sédentaires des plaines quelle est son origine? Pour un Vietnamien ou un Siamois, la forêt est le domaine terrible hautement redouté, des génies malfaisants, des fièvres, des fauves; et les gens capables de vivre dans un tel milieu que peuvent-ils être? sinon des nomades dangereux associés aux bêtes féroces, aux miasmes mortels, aux démons cruels qui errent avec eux dans un tel domaine.

Alors que nous distinguons les nomades des semi-nomades, et de multiples sous-groupes parmi eux, les paysans des rizières et les citadins de tous bords (nés dans le pays ou venus d'Occident) ne voient en eux qu'une masse informe d'être insaisissables, aux réactions inattendues, toujours en mouvement, incapables de s'astreindre à un travail suivi, qui ignorent la valeur de l'argent, etc... Inutile d'évoquer «'idéologie nomade» aux sédentaires, cela les conforterait encore plus dans leurs jugements hautement dépréciatifs dans lesquels le lien très fort des Montagnards à la forêt ennemie de toute "civilisation" pèse le plus. Car les riziculteurs ont peur de la forêt et la première chose que fait un groupe de Vietnamiens ou de Thaïs, c'est d'abattre 
la forêt, mais contrairement aux «primitifs» ils abattent les arbres sans laisser à la végétation le temps de repousser et abîment ainsi le sol qu'ils laissent à découvert. Or, les essarteurs ne subissent pas d'aussi terribles famines qui, chez les sédentaires, tuent des milliers, des dizaines de milliers de personnes; celle de 1945 a fait dans le NordVietnam deux millions de morts et elle ne fut pas la première de cette ampleur rencontrée dans leur histoire. On en a relevé d'équivalentes en Chine et en Inde.

Cependant, malgré cela, les sédentaires des hautes civilisations ont pour idéal de «civiliser» les forestiers, de les réduire à leur standard idéalisé, d'effacer au besoin par la force toute différence avec ce qu'ils considèrent la norme. C'est en présence des effets destructeurs d'une telle politique coercitive que j'ai inventé le terme «ethnocide», calqué sur celui de «génocide», m'étant

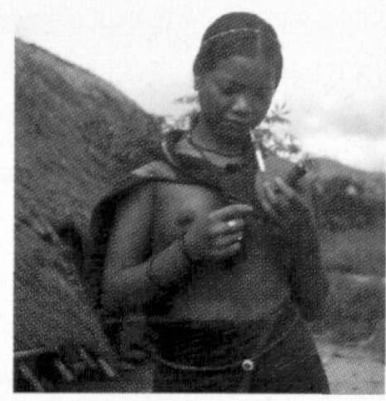
trouvé confronté au profond désarroi des Mnong devant les décisions de feu Ngô Dinh Diêm, alors président de la République du Sud-Vietnam, qui ne supportait pas que les Montagnards eussent des coutumes, des langues différentes de celles des autres habitants du Vietnam, des vêtements différents. Il fallait les forcer à se vêtir de haillons modernes plutôt que de porter les produits de leur magnifique tissage, qu'il condamnait à disparaître. Comme vous pouvez le voir (1984) : «Sar Luk, les travaux et les jours d'un village Mhong Gar du Vietnam central "[?], les Hommes de la Forêt portent de très belles ceintures-tabliers ce qui aux yeux de ce mandarin Washington. ultra-catholique équivalait à la nudité, et la nudité est forcément d'inspiration démoniaque.

Comme on le voit, les civilisés qui en principe ont reçu au moins une teinture scientifique ne comprennent pas que les gens de la forêt font partie du biotope, qu'ils vivent en harmonie avec la faune et la flore, une harmonie que personne n'a intérêt à détruire. Il est vrai que le comportement des civilisés montre qu'ils ne se soucient guère de l'équilibre du milieu. Ainsi, la région où j'ai vécu servait de réserve de chasse à l'empereur Bao Dai. Lui et sa suite claironnaient leur fierté lorsqu'ils avaient abattu, simplement pour le plaisir d'avoir réussi de beaux cartons, plusieurs éléphants, buffles sauvages, tigres, etc... Des massacreurs! Vous avez en France aussi de hauts personnages qui vont en Afrique pour, à leur retour, se vanter d'avoir tué je ne sais combien de lions ou d'autres superbes fauves. Ils détruisent la faune et après cela osent parler d'écologie. Ils osent sortir des décrets, ou plutôt ils poussent les gens au pouvoir qu'ils ont formés à sortir des décrets interdisant de chasser aux indigènes, leurs propres compatriotes restés en symbiose avec leur milieu, alors que ceux-ci, lorsqu'ils chassent ne tuent que pour satisfaire leurs besoins immédiats. Tandis que les puissants des villes ou d'outre-mer, arrivent sur leur terrain avec une provision de boites de conserve, une réserve d'eau non polluée; il y eut même un général américain qui volait des ÉtatsUnis en Centrafrique, avec dans son avion tout un conteneur d'eau potable qu'il apportait de 
Quelle ironie! on ne peut même pas parler de cynisme : ceux qui ont la puissance n'ont conscience que de leur droit. On impose des interdits de chasse aux peuples des forêts dans leur propre habitat, mais ceux qui massacrent les animaux, détruisent la végétation qui gêne leur passage, ces gens des safaris ne sont jamais poursuivis. Mais ce comportement n'est pas l'apanage des seuls Occidentaux : l'administration et ses technocrates luttent contre la mise à feu des essarts nourriciers, mais ferment les yeux sur les destructions commises par les immigrants vietnamiens ou siamois qui, poussés par la guerre ou leur démographie galopante, viennent s'implanter sur les terres des Montagnards : ils abattent les arbres, y compris ceux des bois sacrés, incendient à tort et à travers, exploitent le sol sans aucun ménagement, jusqu'à épuiser sa couche fertile. Dans la région de Saïgon (Ho Chi Minh Ville aujourd'hui), sur la route de Biên Hoa, vous trou-

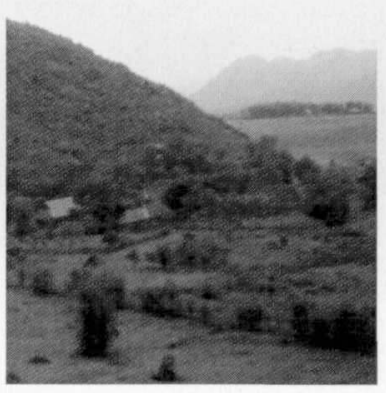
ans de guerre, la déforestation s'est poursuivie, mais alors pour des raisons économiques et à une échelle qui n'a rien à voir avec celle due aux défoliants. L'exploitation industrielle de la forêt ne fait d'ailleurs que reprendre celle que les entreprises américaines ou viétovez de larges étendues de latérite prises sur le domaine des Chrau qui, eux, exploitaient leur finage avec soin depuis des siècles.

Cependant les dégâts dûs aux riziculteurs déracinés n'atteignent pas l'ampleur des destructions commises par les détenteurs de ces technologies de pointe tant admirées. Exemple : l'une des découvertes importantes de la chimie industrielle, celle des défoliants utilisés par les Américains pendant la Guerre du Vietnam. Il y avait dans la région d'An Khê une magnifique forêt : sous la voûte de la haute futaie un enchevêtrement de lianes et d'arbres de taille moyenne cou-

vrant une belle broussaille. J'y suis passé huit ans après l'épandage de défoliants par avion. Tout a disparu, il ne reste qu'une espèce d'arbustes tout petits, malingres, très clairsemés sur un sol rocailleux, stérile. C'est ce que j'appelle un «physiocide» ou un «ecocide». On a tout tué : vous n'avez plus ni arbres, ni plantes comestibles ou autres, ni animaux,... ni hommes.

Comme quoi un progrès technique considérable peut aboutir à une forte régression sur le plan humain. L'écocide d'une importante partie du Vietnam, catastrophique pour ce pays, n'a apporté aucun avantage sérieux aux épandeurs de défoliants. Cependant, la paix revenue après trente

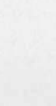


années?... Quelles que soient les critiques qu'on peut en faire, on doit admirer l'espoir inouï dont il était porteur. Ainsi, devant la misère atroce dans laquelle la révolution industrielle avait, à ses débuts, jeté les classes pauvres, les Saint-Simoniens avaient prédit que la machine remplacerait l'homme dans les tâches les plus dures, les plus avilissantes. Ils étaient persuadés que les ouvriers qu'ils voyaient réduits à l'état de brutes par un travail physiquement exténuant, pourraient un jour s'en affranchir et que grâce à la machine, ils finiraient par bénéficier de loisirs jusque là réservés aux seules classes riches, qu'ils pourraient non seulement se reposer, mais surtout se cultiver, s'enrichir intellectuellement.

La vision saint-simonienne du progrès ne peut se réduire à une simple utopie de rêvasseurs impénitents : de même le plus pessimiste doit admettre la réalité d'énormes progrès accomplis dans la condition ouvrière en un

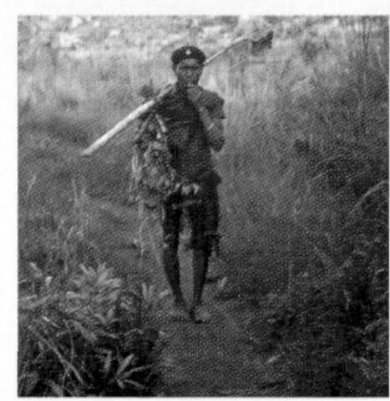
siècle et demi où dans de nom- nuent à la tâche, le grand nombre est rejeté à la marge de la société. Cette hyperproductivité qu'engendre-t-elle en fin de compte : des chômeurs. Des chômeurs dénués de moyens, exclus des avantages acquis par le progrès matériel. Ceux qui travaillent ne disposent pas des loisirs auxquels ils pourraient prétendre s'il y avait un ajustement et une répartition équilibrée du temps de travail qui en outre fournirait au plus grand nombre cette activité vitale minimale, les détachant du désœuvrement humiliant qui pousse à la délinquance.

Lorsqu'on suggère que malgré notre avance technique considérable, on pourrait s'inspirer de certains aspects des sociétés dites «inférieures», cela choque encore beaucoup de gens. Ne touchons pas à la sacro-sainte productivité, mais à la façon d'envisager la vie, ce qui me paraît essentiel en tant qu'êtres humains. On trouve chez nous sous-entendu à notre mythe du breux domaines, la machine a pris la relève de l'homme ou considérablement amenuisé son effort pour un résultat incomparablement supérieur à celui qu'il obtenait autrefois. Mais que constate-t-on actuellement dans les pays industrialisés techniquement les plus avancés? Un terrible blocage. La toute puissance de l'argent exige de produire, produire, et encore produire; et cela à de moindres frais en réduisant les charges sociales, donc le nombre de travailleurs. Au lieu de répartir sur un plus grand nombre, on concentre les tâches sur quelques-uns; les autres de plus en plus nombreux sont laissés pour compte. Les uns s'exté- progrès une conception astreignante, pénible du travail ( «tu mangeras ton pain à la sueur de ton front»). Celle-ci est tout autre chez les Hommes de la Forêt. D'ailleurs le mot «travail» n'existe pas en mnong gar : on décline l'activité que l'on va entreprendre - nourricière, artisanale ou autre par les termes qui la désignent. Lorsque les autorités coloniales ont imposé le travail administratif, il a fallu emprunter à des voisins un terme générique qui s'est chargé de coercition et d'ennui. On considère l'essartage comme étant l'activité la plus importante, celle dont les étapes marquent les jalons de l'année lunaire. On pourrait alors rap- 
procher de notre concept de «travail», par la force à fournir et la fatigue qui en résulte, la courte période d'abattage des arbres et un mois plus tard la mise à feu des abattis suivie de l'étalement des cendres et les semailles, puis quelques mois plus tard, après le sarclage, la moisson. Mais toutes ces activités se font en équipes ce qui constitue autant d'occasions de s'amuser pour les jeunes notamment. En fait, on dispose, le reste du temps, de quantité de journées qu'on emploie à des occupations variées. Les activités individuelles dépendent de la force et de la volonté de chacun, quant aux quêtes de nourriture collectives, elles sont recherchées pour leur côté distrayant. Ainsi, lors des parties de cueillette de plantes sauvages comestibles, la plupart des hommes vont chasser ou pêcher individuellement ou à deux ou trois, les femmes se rassemblent en larges groupes, elles s'interpellent «Viens, Une Telle, viens on va cueillir des gnetum, on va

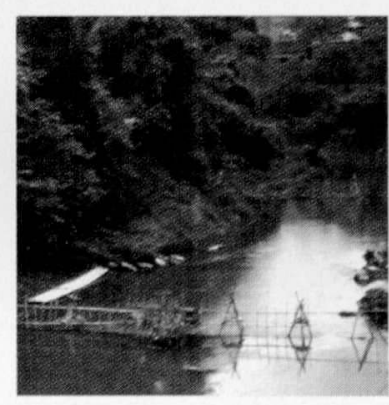
s'amuser (dlang ber)!». On part en forêt en plaisantant, en chantant (remarquez que le chant et les rires servent aussi à écarter le tigre). Un homme marche en tête armé de son coupe-coupe, un autre clôt la file indienne, lui aussi armé de sa machette. Arrivés sur le terrain de cueillette indiqué par des vadrouilleurs, on récolte juste ce qu'il faut pour la consommation raisonnable de chaque famille; on revient dans la même ambiance, car on ne s'est pas crevé à la tâche. On ménage ses propres forces et on ne dilapide pas les ressources du milieu qui entretient la vie du groupe. De même pour la chasse et la pêche : on ignore le massacre du gibier par vanité ou désir d'enrichissement, d'ailleurs toute grosse prise donne lieu à partage.

Ce respect de l'environnement (que pour être à la mode, on pourrait appeler, un sens de l'écologie, mais sans programme), cette solidarité qui rend distrayantes les activités de production et toute acquisition importante profitable à tous les membres de la collectivité y compris les moins doués ou les moins chanceux. Il me semble que tout cela mériterait de retenir l'attention et servir de source d'inspiration. Certains vont me prendre pour un vieux conservateur prônant le retour aux vieilles traditions et pourquoi pas, par nostalgie, une rétrogradation à la chandelle, à la loupiotte de bois résineux. Non, bien au contraire, ce qui me préoccupe en tant que père et citoyen, c'est la situation actuelle et l'avenir des sociétés industrielles dans lesquelles nous vivons. Après les fantastiques destructions causées à la surface de la planète Terre au nom du Progrès, on a pris un peu tard conscience de la nécessité de respecter l'équilibre des écosystèmes, alors que les «sauvages» eux le font naturellement depuis toujours et sans discours. Quand je dis «on», j'emploie une figure de style : ce «on» regroupe bien peu de monde et pèse si peu dans les décisions que les destructeurs, qui eux détiennent le pouvoir, poursuivent en Amazonie, en Indonésie et ailleurs leurs entreprises néfastes et pour les indigènes de ces régions et pour l'ensemble de l'humanité, ce qui comprend d'ailleurs les destructeurs eux-mêmes.

Un autre sujet très inquiétant, qui a fini par secouer l'opinion et toucher toutes les classes 
sociales, c'est celui du chômage et de l'exclusion. La société a tiré un faible profit relatif des avantages considérables apportés entre autres par la machine : au contraire, ainsi que je viens de le souligner, on voit se réduire comme peau de chagrin le nombre de travailleurs et aujourd'hui tous les pays anciennement ou récemment industrialisés jettent sur le pavé des quantités de plus en plus grandes d'exclus, de miséreux. Le développement général spectaculaire de l'enseignement, et en particulier de l'enseignement supérieur, a permis à un nombre accru de jeunes d'éclore leurs dons qui sans. lui seraient restés amorphes et de diversifier leurs loisirs, ne serait-ce que sur le plan culturel; malheureusement, la réduction malthusienne des débouchés, les empêche de profiter de cet enrichissement et les plonge dans l'insatisfaction et le désarroi. Pourquoi ne pas réexaminer en profondeur la conception du travail, atténuer la précipitation et la surenchère et lui insuffler cet aspect ludique que revêtent chez les Gens de la Forêt, les activités nécessaires à l'homme pour vivre. Réveiller le goût du don, de l'échange chers à Mauss, que notre société tend à négliger, alors qu'ils sont indispensables à l'homme en société.

Nous sommes en fait englués dans une conception privilégiant à outrance la thésaurisation égoïste et qui rend incapables la grande majorité d'entre nous d'apprécier chez les «primitifs» la sagesse de leurs règles d'harmonie sociale. Je n'exagère en rien. Vous avez sans doute vu des photos de l'extraordinaire Uluru, l'Ayers
Rock des cartes. Ce colossal monument naturel à dominante rouge qui se dresse au centre du désert australien attire un grand nombre de touristes. Un matin où je n'ai pu escalader le rocher, j'ai, par oisiveté et curiosité, suivi un groupe de touristes allemands et hollandais. Des gens apparemment riches car le voyage d'Europe à Sydney coûte très cher, et de Sydney à Ayers Rock également. Leur guide, un ranger, leur expliquait les peintures d'une grotte, ce qui l'a entrainé à livrer quelques détails sur le genre de vie aborigène. Cet auditoire très attentif a montré encore plus d'intérêt lorsque, en bon professionnel de la communication, le ranger a déclaré que les Aborigènes étaient de «very strange fellows». Puis, sans doute inspiré par sa longue expérience des touristes, il appuie son propos d'un solide exemple : lorsqu'un Aborigène tue un kangourou à la chasse, il ne garde pas sa victime pour lui seul, mais partage avec les autres le gibier qu'il a lui-même tué et n'en reçoit qu'une part. Étonnement délirant de ce groupe de gens repus et couverts de bijoux et en principe, grâce à leur fortune, un minimum cultivés. Pas une seule voix pour admirer l'esprit d'abnégation et de solidarité des Aborigènes, mais d'abondantes questions du type : "Le chasseur partage-t-il toujours le gibier qu'il a tué?». Stupeur encore plus grande lorsque vient la réponse : «Ce n'est pas lui qui effectue le partage, il ne fait que recevoir une part!».

Et pourtant il s'agit là de règles archiconnues des ethnologues et qui, à nos yeux, vont de soi. L'étonnement de ces touristes nous sur- 
prend, mais n'oublions pas que nous avons eu la chance de vivre chez les gens de la forêt, de partager leur vie quotidienne ponctuée de rituels, et que nous en sommes revenus imprégnés. Car l'ethnologie a ceci de particulier qu' elle est autant un genre de vie qu'une discipline scientifique. La difficulté du réajustement, au retour, vient peutêtre de ce qu'on a quitté un quotidien chargé de poésie (on y a découvert que celle-ci était aussi nécessaire que le pain ou le riz chez les peuples matériellement les plus démunis), pour le quotidien qu'on avait fui et qui la relègue à des spécialistes et la réduit en fait à une affaire individuelle.
NOTE

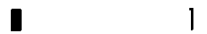

Voir filnoyraphie en fin de volume. 